\title{
Diderot Studies, tome XXXIV
}

\section{Eszter Kovács}

\section{(2) OpenEdition}

\section{Journals}

\section{Édition électronique}

URL : http://journals.openedition.org/studifrancesi/10578

DOI : 10.4000/studifrancesi. 10578

ISSN : 2427-5856

\section{Éditeur}

Rosenberg \& Sellier

\section{Édition imprimée}

Date de publication : 1 décembre 2017

Pagination : $551-552$

ISSN : 0039-2944

\section{Référence électronique}

Eszter Kovács, «Diderot Studies, tome XXXIV », Studi Francesi [En ligne], 183 (LXI | |II) | 2017, mis en ligne le 01 août 2018, consulté le 23 janvier 2021. URL : http://journals.openedition.org/studifrancesi/ 10578 ; DOI : https://doi.org/10.4000/studifrancesi.10578

Ce document a été généré automatiquement le 23 janvier 2021.

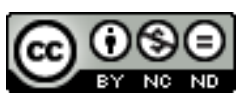

Studi Francesi è distribuita con Licenza Creative Commons Attribuzione - Non commerciale - Non opere derivate 4.0 Internazionale. 


\title{
Diderot Studies, tome XXXIV
}

\author{
Eszter Kovács
}

\section{RÉFÉRENCE}

Diderot Studies, tome XXXIV, éd. par Thierry BELLEGUIC, Genève, Droz, 2014, 386 pp.

1 Le volume XXXIV des Diderot Studies, daté de 2014, mais en fait disponible depuis l'année dernière, réunit vingt-et-un articles, dont certains sont consacrés à des sujets peu connus même par les diderotistes. On trouve six-sept articles dans le dossier thématique «Diderot et le supplément» et quatre articles dans les «Miscellanées». Les éditrices du dossier thématique sont Kate Tunstall et Caroline Warman.

Thierry BELLEGUIC se donne la tâche de définir la problématique dans l'article D'un grain l'autre. Sur Diderot et le supplément (pp.5-39): il examine comment Diderot écrit en suppléant ou, autrement dit, comment il supplée en écrivant. L'article qui ouvre le volume fait l'impression d'une «promenade dans l'œuvre» pour illustrer l'originalité de cette manière de penser, si propre à Diderot. Michel DELoN, dans l'article Un matérialisme de la note (pp.41-52), examine l'annotation en tant que mode d'écrire: il s'agit pour Diderot, comme pour Bayle, d'étendre le texte et d'élargir la réflexion. Diderot annote les textes des autres, ses propres textes et il est lui-même annoté: M. Delon nous présente une édition de Venise des Pensées philosophiques (1754) et montre que le fait d'annoter est également l'ouverture d'une discussion.

3 Nathalie FERRAND, dans l'article Illustrer Diderot (pp.53-70), propose d'examiner les illustrations des romans de Diderot, plus particulièrement celles de la première édition des Bijoux indiscrets. L'article tente de nous montrer que ces images peuvent en vérité «illustrer» le texte. James FOWLER, dans Diderot's Anxiety of Influence (pp. 71-88), applique une idée-force de Harold Bloom dans son ouvrage The Anxiety of Influence: comment faire preuve de son talent? Il examine ce phénomène à travers le rôle de Richardson chez Diderot: comment égaler l'auteur anglais, voire même le surpasser? En quelque sorte, La Religieuse en sera l'essai. Dans Un géomètre embarrassé dans des toiles d'araignée (pp. 89-101), Catriona SETH fait connaître un aspect moins connu de l'œuvre de Diderot: 
l'écrivain prend aussi part aux discussions mathématiques dans la Correspondance littéraire. Diderot s'oppose à D'Alembert et affirme que l'inoculation ne doit pas être refusée en suivant des calculs de probabilité d'effets bénéfiques ou nocifs.

Dans Théorie et pratique de la langue (pp.103-120), Élise PAVY-GUILBERT analyse le préambule du Salon de 1767 en tant qu'addition programmée à la Lettre sur les sourds et muets. Le discours critique sur les tableaux s'émancipe pour focaliser sur le langage: le texte devient ainsi une démarche autoréflexive. Comme le remarque É. Pavy-Guilbert, le langage doit rivaliser avec l'image pour Diderot, ce qui est un enjeu essentiel pour le critique d'art. L'article Le philosophe masqué et la chimère (pp. 121-132) de Frank CABANE est centrée sur l'Addition aux Pensées philosophiques, un texte que la recherche redécouvre. Diderot entreprend cette Addition à propos des Objections diverses contre les écrits de différents théologiens, dont il radicalise les arguments. Ce mode d'écriture témoigne d'un déplacement du déisme vers l'athéisme: l'analyse de F. Cabane convainc son lecteur du rôle que l'Addition joue dans l'évolution de la pensée de Diderot. L'article Diderot et le langage médical d'Alexandre WENGER (pp. 133-144) montre que la médecine apparaît dans d'autres textes de Diderot que ceux que l'on évoque le plus souvent à ce propos, notamment dans Mystification, à côté de l'exemple plus connu du Rêve de D’Alembert. De toute évidence, «le médical» forme un réseau dans la pensée de Diderot et l'examen de cet aspect peut être approfondi au-delà des cadres d'un seul article.

Jean-Claude BouRDIN s'occupe de L'auto-citation dans le "Neveu de Rameau" (pp. 145-184). Il s'agit d'un texte de Diderot si souvent analysé et commenté (en diverses langues) qu'il devient difficile d'en écrire sans être angoissé par la quantité de la littérature critique existante. J.-Cl. Bourdin présente Le Neveu en tant qu'une quintessence de la pensée subversive de Diderot. En raison de la grande variété des analyses, on a tout de même l'impression que l'étude sort du thème désigné dans le titre pour proposer une interprétation plus complexe de l'ouvrage. Diderot tend à occulter ceux dont il annote et commente le texte. Dans son article La dynamique matérielle d'après les "Observations sur Hemsterhuis” (pp. 185-198), Colas DufLo reconsidère les valeurs d'Hemsterhuis, dont les idées permettent à Diderot de reformuler et confirmer sa pensée matérialiste à partir d'une réflexion anti-matérialiste.

6 L'article de Russel Goulbourne, Diderot and Horace: from Translation to Imitation (pp. 199-214), est instructif car Diderot réfléchit sur un concept très en vue en traductologie contemporaine, notamment l'intraduisibilité. Or, la réflexion de Diderot, malgré son originalité, est rarement évoquée par les traductologues de nos jours. Dans $\mathrm{Du}$ "Voyage" de Bougainville au "Supplément" de Diderot (pp. 215-226), Isabelle MOREAU choisit un thème relativement bien connu par les diderotistes mais toujours intéressant. On regrette un peu que, après des analyses tout à fait pertinentes sur Bougainville et le regard sur son rôle en tant que voyageur, la partie consacrée au Supplément de Diderot soit trop concise. Flore VILLEMIN-DE CARRÉ, dans Analyse de la réfutation dans "Le Rêve de D’Alembert" (pp. 227-243), présente la réfutation en tant qu'élément discursif et argumentatif, une facteur opératoire du dialogue.

7 L'identification des contributions de Diderot à l'Histoire des deux Indes est une enquête passionnante et on doit toujours remercier les grands spécialistes, tels Gianluigi Goggi et Antony Strugnell, de partager leurs expériences avec les générations de chercheurs plus jeunes. Dans Diderot's Unattributed Contributions to the "Histoire des deux Indes" (pp. 245-257), Antony STRUGNELL nous propose, à côté des concordances thématiques, l'analyse stylistique en tant que critère d'examen. La démarche mérite d'être 
considérée comme une méthode à part entière, surtout parce qu'il y a quelque chose de particulièrement unique dans l'écriture diderotienne que Raynal veut garder justement pour son efficacité rhétorique. Dans La réflexion morale de "L'Essai sur Sénèque" (pp. 259-268), Charles VINCENT tente de saisir l'essentiel du dernier ouvrage de Diderot. Est-ce possible? En tous cas, l'article réussit à faire plusieurs constatations de première importance pour situer cette œuvre polycentrique, ce qui est d'autant plus difficile que l'Essai tend à s'échapper aux tentatives de catégorisation.

8 L'article d'Angelica GOODDEN, Bouquets and the Blind (pp. 269-282), se concentre en effet plus sur Rousseau que sur Diderot, mais examine une question également au cœur de leur pensée, notamment le rapport entre les sens. Caroline WARMAN, dans Naigeon, éditeur de Diderot physiologiste (pp. 283-302), examine comment Naigeon se sert du Rêve de D'Alembert et des Éléments de physiologie dans ses Mémoires. Selon son analyse, le rôle de Naigeon dans la médiation de ces textes est plus important qu'on ne le considère en général. En fait, C. Warman refait cet examen de manière plus précise, grâce aux versions électroniques et un nouvel intérêt pour ce sujet: ce type de travail, un peu ingrat à faire, est toujours précieux pour la communauté scientifique.

9 Parmi les Miscellanées, on trouve l'article de John PARKIN, Comic Patterns in "Le Neveu de Rameau" (pp. 305-316), ce qui est un sujet riche tant Diderot exploite toutes les facettes du comique dans le Neveu. L'article de Florence viDonNE, Diderot par Greuze (pp. 317-330) étudie un moment particulier dans «l'iconographie» de Diderot: le dessin par Greuze de Diderot sur son lit de mort. Jean-Christophe REBEjKow, dans Le Dilemme de Diderot (pp.331-352), analyse la Lettre apologétique en cherchant les analogies, les points communs avec l'Essai sur Claude et Néron. S'agit-il d'une mystification dans la Lettre apologétique? À mon avis, tout au plus d'une mystification qui n'a jamais effectivement trompé personne. Il faut également noter qu'Éric Gatefin a consacré un article exactement au même sujet (Revue Épistolaire, 2015), ce dont, évidemment J.C. Rebejkow n'a pas pu avoir connaissance, vu les dates de parution des deux articles. Dans What can the Possessed Possess? (pp.353-376), Lorraine PIRoux note une contradiction apparente entre la créativité ou l'originalité et le contrôle de soi nécessaire à faire une œuvre d'art. Le génie qui exerce un contrôle sur soi est en effet l'acteur doué. L. Piroux fait l'analogie entre l'estime de l'acteur et l'estime de l'auteur (dans le sens moderne de ce dernier terme).

Ce nouveau volume des Diderot Studies intéressera à juste titre les chercheurs. Les sujets sont assez variés, le volume est bilingue et on ne peut que souligner l'heureux équilibre des générations: les rédacteurs offrent une possibilité de publier aux chercheurs aux différents stades de leur parcours professionnels. Je voudrais pourtant remarquer que l'ordre des articles dans le dossier thématique ne me paraît pas toujours logique, même s'il aurait été presque impossible de les arranger de manière tout à fait conséquente. 